\title{
Word and nonsense anagram solution with tachistoscopic stimulus presentation'
}

\author{
HARRY BEILIN AND HAROLD L. LEVINE \\ BROOKLYN COLLEGE, THE CITY UNIVERSITY OF NEW YORK
}

Word and nonsense anagram stimuli were tachistoscopically presented ( $1 \mathrm{sec} ., 1 / 2 \mathrm{sec}$.), in two presentation orders, to four groups totalling $80 \mathrm{Ss}$. Word anagrams took longer to solve than nonsense anagrams. There was also a significant interaction between exposure time and presentation order. The results are interpreted as supporting a cognitive analysis of anagram problem solving.

In prior studies (Beilin \& Horn, 1962; Beilin, 1966; Beilin, in press), it was shown that anagram solution time was longer for word anagrams than nonsense anagrams. It was inferred that word anagrams are more difficult to solve because the organizational properties of the word provide a structure more difficult to reorganize than with nonsense arrangements. As is typical in anagram problem solving studies, stimulus exposure time was fairly long (i.e., 2 min.). If exposure time were to be reduced, however, a new set of conditions would exist which could conceivably affect the solution processes. With short (tachistoscopically controlled) duration, problem solving would be contingent upon verbal memory or visual imagery. Two opposing tendencies should then operate. Word anagrams should be easier to solve because a word image would be easier to retain or remember than a nonsense arrangement. On the other hand, since the organizational properties of words make them more difficult to reorganize than nonsense arrangements, word anagram solution should remain more difficult in spite of the ability to recall them more easily. The present study was designed to test these possibilities.

\section{Method}

Design. The principal effects studied were Exposure Time (1 sec. vs. 1/2 sec.), Presentation Order (word anagrams first, nonsense anagrams second vs. nonsense anagrams first, word anagrams second) and Anagram Type (word anagrams vs. nonsense anagrams). Solution time was the dependent variable. There were four groups of Ss. Each S solved the words and nonsense anagrams with the order determined by the group to which he was randomly assigned (Table 1). There were $20 \mathrm{Ss}$ in each group.

Table 1. Mean anagram solution time with two stimulus exposures and two presentation orders. ( $N=20$ in each group)

\begin{tabular}{lcccc} 
Order & \multicolumn{2}{c}{ Word Anagrams } & \multicolumn{2}{c}{ Nonsense Anagrams } \\
& 1 sec. & $1 / 2$ sec. & 1 sec. & $1 / 2$ sec. \\
\hline First & 19.65 & 27.65 & 19.82 & 17.20 \\
Second & 27.52 & 19.14 & 11.04 & 19.93 \\
\hline
\end{tabular}

Materials. The stimuli consisted of the same set of 10 five-letter word anagrams and 10 nonsense anagrams we have previously used (Beilin \& Horn, 1962). Each anagram was solved by interchanging only two letters.

The anagrams were projected on a 9 in. $x 9$ in. Sawyer viewing screen by means of a Kodak Carousel Projector (Series 800) equipped with a Wollensak tachistoscopic lens attachment. When on the screen, the stimulus letters were spaced about $1 / 4$ in. apart and measured approximately $1 / 2$ in. high. The $S$ sat about $3 \mathrm{ft}$. from the screen.

The exposure times were chosen on the basis of a pilot study with another $20 \mathrm{Ss}$, in which the stimulus was identified by pronouncing the word or spelling the nonsense arrangement after exposure. Particular recall difficulty with two nonsense anagrams was also revealed. When presentation of these stimuli was preceded by instructions to pay particular attention to these stimuli, they were then easily recalled. This procedure was carried over to the experiment itself. Later examination revealed response to these anagrams to be no different from others in in the series.

Procedure. Instructions were the same as given in our prior studies except that anagram solutions were not given for unsolved anagrams. There was a $1 \mathrm{~min}$. maximum solution time. At the completion of the 20 test trials, the $S$ was questioned as to his method of solution and asked to identify the "easier" problem.

Subjects. Eighty Brooklyn College sophomore and junior students were individually tested.

\section{Results}

Two scores were available for each $\mathrm{S}$, his median word anagram solution time and his median nonsense anagram solution time. The mean of these medians was computed for each of the four experimental groups (Table 1).

A 2 by 2 by 2 analysis of variance for one repeated measure was performed on the data (Winer, 1962). The Anagram Type main effect was significant, with word anagram solution time significantly longer than nonsense anagram solution time (Word mean $=23.49$, Nonsense mean $=17.01 ; p<.001)$. This supports prior findings of Beilin \& Horn (1962), Ekstrand \& Dominowski (1965), Beilin (1966), and Beilin (in press).

The only other significant effect was the Exposure Time by Presentation Order interaction $(p<.05)$. For this analysis the word and nonsense anagram solution times were averaged. The means for the word-nonsense groups were: 1 sec. $-15.34 ; 1 / 2$ sec. -23.78 . Means for the nonsense-word groups were : 1 sec.-23.66; $1 / 2$ 
Table 2. Relation of ascribed anagram solving difficulty to actual difficulty.

\begin{tabular}{lccc} 
Ascribed Difficulty a & \multicolumn{2}{c}{ Performance } \\
& $\begin{array}{l}\text { Word } \\
\text { Anagrams } \\
\text { Easier }\end{array}$ & $\begin{array}{l}\text { Nonsense } \\
\text { Anograms } \\
\text { Easier }\end{array}$ & Total \\
\hline W A Eosier & 21 & 12 & 33 \\
N A Easier & 7 & 27 & 34 \\
Total & 28 & 39 & 67 \\
\hline
\end{tabular}

a $9 \mathrm{~S}$ s saw no difference; $4 \mathrm{~S}$ s gave ambiguous responses. $\chi^{2}=11.05, d f=1, p<.001$.

sec.-18.19. An examination of the components of these scores helps clarify the interaction. With the longer (1 sec.) exposure time, when word anagrams are solved first, mean solution time for the nonsense anagrams which follow is shorter than the reverse, indicating positive transfer from word anagram solution, and negative transfer from nonsense anagram solution, although, when presented first, word anagrams take longer to solve in the $1 / 2 \mathrm{sec}$. than the $1 \mathrm{sec}$. condition, whereas the reverse is true for nonsense anagrams presented first. Looked at another way, in the $1 \mathrm{sec}$. condition, when word anagrams are solved first, solution time is shorter than when they are solved second; in the $1 / 2 \mathrm{sec}$. condition the reverse is true. Again, in the 1 sec. condition when nonsense anagrams are first, solution is longer than when second; in the $1 / 2 \mathrm{sec}$. condition the reverse is true.

Table 2 presents the relationship between the S's judgment of anagram difficulty and his actual performance difficulty. The relationship is significant $(p<$ $.001)$, indicating substantial accuracy in judgment. At the same time, while $49 \%$ of the Ss who responded appropriately thought word anagrams were easier, $28 \%$ of these Ss' performance did not agree with their judgments. For about $65 \%$ of the total group, the nonsense anagrams were actually easier to solve. All of the nine who perceived no difference in difficulty, in fact, performed better on the nonsense anagrams. Most of the Ss who said words were easier, attributed this to the fact that they could remember the letters of the words more readily. Those who said nonsense anagrams were easier, mentioned that it was more difficult to rearrange the letters of a meaningful word than of a nonsense arrangement.

\section{Discussion}

In spite of the fact that reduced exposure time places a greater burden on a S's memory and imagery, which presumably adds an advantage to word anagram solution, word solution time is still significantly longer than nonsense anagram solution time. There is, additionally, a somewhat greater belief among Ss of the ease of word anagram solution than is reflected in performance $49 \%$ vs. $42 \%$ ). These data lead to the conclusion that the organization of words acts as a powerful agent in overcoming the effects of memory and imagery, although it is also possible that organization effects and memory combine to make word anagrams more difficult to solve.

Positive transfer from word anagram solution to nonsense anagram solution indicates that the reorganization of well organized units makes it easier to reorganize less-well structured stimuli that follow, whereas the reorganization of less-well organized units inhibits the solution of well organized units that follow. These organization effects interact with exposure time effects, presumably memory and imagery, to create inverse relations among solution times. In contrast to this, with extended exposure time (2 min.) there was a slight transfer effect, but opposite to the one reported here, i.e., word solution was somewhat shorter after nonsense solution and nonsense solution somewhat longer after word solution. These effects were nonsignificant, however (Beilin \& Horn, 1962).

This study, as well as supporting an organizational interpretation of anagram problem solving, demonstrates the experimental utility of tachistoscopic control of anagram problem solving processes.

\section{References}

Beilin, H. Solving words as anagrams: A re-examined issue examined. Psychon. Sci., 1966, 6 (2),

Beilin, H. Developmental determinants of word and nonsense anagram solution. J. verbal Learn. verbal Behav., (in press).

Beilin, H., \& Hom, R. Transition probability effects in anagram problem solving. J. exp. Psychol., 1962, 63, 514-518.

Ekstrand, B. G., \& Dominowski, R. L. Solving words as anagrams. Psychon. Sci., 1965, 2 (8), 239-240.

Winer, B. J. Statistical principles in experimental design. New York: McGraw-Hill, 1962.

\section{Note}

1. This study was supported in part by National Institute of Child Health and Human Development Research Grant HD 00925-05 to the senior author. Mr. Levine participated as part of a Masters seminar. 